\title{
Penser la flexicurité au niveau « méso » : entre ancrage territorial et innovation sociale
}

\section{Developing flexicurity at a meso level: between local roots and social innovation}

\author{
Anne Cromarias \\ Centre de Recherche Clermontois en Gestion et Management (CRCGM) \\ Groupe ESC Clermont, 4, Bd Trudaine, 63037 Clermont-Ferrand Cedex
}

\section{Résumé}

La flexicurité, concept à la fois paradoxal, ambigu et à fort contenu idéologique, tente de proposer des éléments de réponse à un marché du travail écartelé entre des exigences de flexibilité accrues pour les entreprises et une précarité (réelle ou potentielle) de plus en plus marquée pour les salariés. L'objet de cet article est de s'intéresser à l'opérationnalisation de la flexicurité. Sa thèse est que, compte tenu de la place aujourd'hui accordée à l'organisation réticulaire des entreprises, d'autant plus si l'on considère les petites et moyennes organisations, l'échelon «méso » semble le plus pertinent.

En s'appuyant sur une étude de cas multi-sites portant sur quatre terrains permettant ainsi de varier les contextes, les acteurs et les pratiques visées, l'article montre que les pratiques effectives de flexicurité s'appuient à la fois sur l'ancrage territorial des acteurs, au sens d'une proximité géographique doublée d'une proximité organisée, et sur l'ambition de proposer des réponses effectives à des problématiques sociales et sociétales fondamentales. Les différents acteurs de la flexicurité agissent dans et par un milieu qui s'avère déterminant pour la réussite des pratiques observées.

(c) 2010 Lavoisier, Paris. Tous droits réservés.

\footnotetext{
*Adresse email : anne.cromarias@esc-clermont.fr doi:10.3166/ges.12.415-435 @ 2010 Lavoisier, Paris. Tous droits réservés.
} 


\section{Summary}

As a concept, flexicurity is all at once paradoxical, ambiguous and strongly tinted with ideology since it tries to help provide answers in a work market torn between firms' growing need for flexibility and the actual or potential rise of precariousness for the employees. The present article focuses on how to make flexicurity work. It purports to show the prime importance of the meso level, considering the precedence of the reticular structure in firms nowadays, particularly SMOs.

Through a case study of four local sites providing a variety of actors, contexts and purposes, the article shows that, to be effective, flexicurity practices have to be rooted both in the stakeholders local background, implying geographic and organizational proximity, and in their will to provide genuine answers to vital social and societal problems. The various promoters of flexicurity exercise their role thanks to the local background which emerges as the key to make flexicurity a real winner.

(c) 2010 Lavoisier, Paris. Tous droits réservés.

Mots clés : flexicurité, territoire, proximité, « gagnant-gagnant », collaboration.

Keywords : flexicurity, local background, proximity, win-win, collaboration.

À mi-chemin entre politique macro-économique et théorie du travail et de l'emploi, la flexicurité est un concept à la fois paradoxal, ambigu et à fort contenu idéologique. Directement issue des exemples hollandais et danois, la flexicurité tente de proposer des éléments de réponse à un marché du travail écartelé entre des exigences de flexibilité accrues pour les entreprises et une précarité (réelle ou potentielle) de plus en plus marquée pour les salariés.

L'objet de cet article est de s'intéresser à l'opérationnalisation de la flexicurité. Sa thèse est que, compte tenu de la place aujourd'hui accordée à l'organisation réticulaire des entreprises, d'autant plus si l'on considère les petites et moyennes organisations, l'échelon « méso » semble le plus pertinent. Pour cela, nous nous appuyons sur une étude de cas multi-sites, mobilisant les outils de l'étude qualitative. L'investigation porte sur quatre terrains, permettant ainsi de varier les contextes, les acteurs et les pratiques visées, deux cas pouvant être qualifiés de « réussites » et les deux autres pouvant être considérés comme des « échecs », puisque, parmi les pratiques de flexicurité effectivement observées, certaines fonctionnent et d'autres ont avorté.

Après avoir précisé le concept de flexicurité et justifié l'intérêt de considérer le niveau méso-organisationnel (partie 1), nous présenterons la méthodologie d'intervention et les quatre cas étudiés (partie 2). Nous proposerons alors des pistes de réflexion pour situer les pratiques de flexicurité entre ancrage territorial et innovation sociale (partie 3).

\section{Pourquoi une flexicurité au niveau « méso » ?}

\subsection{La flexicurité, un concept en construction}

La flexicurité est un concept en construction, le terme, non stabilisé en langue française ${ }^{1}$, ayant été utilisé pour la première fois dans les années 1990 par le Ministre du Travail hollandais. Cet oxymore, associant deux termes a priori inconciliables, est qualifié

\footnotetext{
${ }^{1}$ Les appellations « flexi-sécurité », «flexécurité » sont également utilisées, mais nous leur préférons celui de « flexicurité », qui semble aujourd'hui être le plus utilisé.
} 
par Barbier (2009) de « chat - chien typique ». La notion a été rapidement utilisée dans les travaux académiques, pour s'intéresser d'une part aux politiques à l'œuvre aux PaysBas en vue d'harmoniser les droits sociaux des différents contrats de travail, notamment les plus atypiques (flex-jobs), la flexicurité étant ici issue d'une longue négociation entre partenaires sociaux (Wilthagen \& Rogowski, 2002 ; Wilthagen \& Tros, 2003) et, d'autre part, au « triangle d'or » danois, caractérisé par une grande flexibilité du marché du travail, une protection sociale élevée et des politiques actives de l'emploi (Jørgensen \& Madsen, 2007), aboutissant au modèle danois de flexicurité, caractérisé notamment par un contexte historico-économico-culturel tout à fait particulier (Madsen, 2004 ; Bredgaard et al., 2005 ; Boyer, 2006).

La flexicurité peut être représentée de façon matricielle par des échanges (trade-offs) entre quatre formes de flexibilité (numérique interne ou externe, fonctionnelle interne ou externe) et quatre formes de sécurité (du poste, de l'emploi, de revenu ou d'option) (Leschke et al., 2006). Elle est par ailleurs inscrite depuis 2005 comme l'une des lignes directrices pour l'emploi de la Stratégie Européenne pour l'Emploi, à travers des principes communs libellés en quatre composantes : le contrat de travail avec « des dispositions contractuelles souples et sûres », la formation ou « apprentissage tout au long de la vie », les politiques de l'emploi ou «politiques actives du marché du travail » et la protection sociale, c'est-à-dire des « systèmes de sécurité sociale modernes $»^{2}$.

Cependant, cet apparent consensus ne doit pas cacher toute l'ambiguïté du concept, notamment sur le plan des représentations de la part des principaux acteurs directs (qui expérimentent au quotidien la flexicurité : les employeurs et leurs salariés) ou indirects. En France, la connotation idéologique du terme est tellement marquée que les partenaires sociaux, ceux-là mêmes qui l'ont défendue et négociée aux Pays-Bas ou au Danemark, osent à peine s'en emparer, préférant le terme de " sécurité sociale professionnelle » (Cahuc \& Kramarz, 2004) ou de « sécurisation des trajectoires » (Méda \& Minault, 2005) 3 .

Les enjeux de la flexicurité sont néanmoins clairs : il s'agit d'apporter simultanément de la flexibilité et de la sécurité, aux employeurs comme aux salariés, selon le principe de « gagnant - gagnant ». Pour les entreprises, l'intérêt essentiel est de permettre un ajustement, dans un délai le plus court possible, de la main-d'œuvre disponible, en termes de qualité (compétences) et de volume (effectif). Cependant, l'enquête « Acemo Flexibilité » menée en 2000 par la DARES apporte des éclairages complémentaires. Si, sans surprise, la probabilité de recourir à un mode de flexibilité dépend largement de la taille de l'entreprise, de son secteur d'activité, des caractéristiques de la main-d'œuvre et des fluctuations de l'activité, l'analyse économétrique fine des données de l'enquête donne des résultats plus mitigés quant à l'arbitrage qui peut être fait entre les différents modes de flexibilité, montrant que les pratiques de flexibilité dans les entreprises sont multiformes et ne semblent pas toujours obéir à une logique extérieure, modélisable et identifiable. Ces pratiques relèveraient plutôt de choix stratégiques - peut-être aussi d'habitudes (Bunel, 2004). Il est par ailleurs fréquent de considérer le recours à la flexibilité, surtout externe, comme faisant des

\footnotetext{
${ }^{2}$ Source : Union Européenne ; http://ec.europa.eu/social/main.jsp?catId=117\&langId=fr

${ }^{3}$ En France, la flexicurité fait l'objet d'un débat passionné depuis le milieu des années 2000. L'accord du 11 janvier 2008 sur la modernisation du marché du travail est considéré par certains comme une amorce de flexicurité, notamment du fait du dispositif de «portabilité de certains droits » pour les demandeurs d'emploi. Si cette dimension politique ne saurait être occultée, elle n'est cependant pas abordée dans le présent article.
} 
victimes : les salariés. Cette image d'Epinal recouvre une réalité certaine. Qu'il s'agisse du temps partiel, du CDD ou de l'intérim, ces situations sont loin d'être systématiquement un choix de vie de la part des salariés concernés. D'ailleurs, les trois-quarts des salariés sous contrat temporaire auraient préféré obtenir un CDI (Fabre \& De Riccardis, 2007). Inversement, pour un salarié sur cinq concernés par un emploi temporaire, il s'agit d'un choix. Cette population trouve au moins deux avantages à sa situation : temps libre entre deux contrats et liberté accrue dans l'entreprise du fait d'un moindre engagement.

Mais la principale difficulté à cerner la réalité, au-delà des différences de situation évidentes, tient au fait que l'on se base sur des perceptions, qui recouvrent elles-mêmes des conceptions éventuellement très différentes. Ainsi précarité de l'emploi n'est pas synonyme de précarité du travail. De même précarité du travail ne rime pas nécessairement avec précarité des conditions d'exercice du travail. S'ajoute à cela la subtilité entre les termes d'insécurité et d'instabilité, qu'il s'agisse du travail ou de l'emploi. Pour le CERC (2005), la stabilité de l'emploi définit la continuité du lien d'emploi entre un salarié et une même entreprise, même en cas de changement de statut du contrat. Quant à la sécurité de l'emploi, elle désigne le fait, pour une personne, de demeurer employée sans interruption notable, même s'il y a changement d'entreprise. On comprend aisément que si l'on ne parle pas des mêmes choses, il est difficile de s'entendre. En ajoutant à ces ambiguïtés de termes une perception, nécessairement subjective, demandée aux salariés interrogés, il semble très délicat de prétendre mesurer de façon fiable le ressenti des salariés.

L'employabilité, comprise comme "la capacité à demeurer autonome dans un développement de carrière, la capacité à intéresser successivement plusieurs employeurs, à changer de voie, à forger son itinéraire professionnel »(Gazier, 2003), semble un concept central puisque, compte tenu des évolutions socio-économiques, l'individu est de plus en plus maître de son propre parcours. La flexicurité peut dès lors se concevoir comme l'un des instruments qui, selon le cas considéré, aide ou oblige l'individu à développer sa propre employabilité. Inversement, on peut aussi considérer la flexicurité comme une cible, qui ne peut être atteinte qu'en améliorant l'employabilité des individus. Moyen ou résultat, flexicurité et employabilité sont donc étroitement liées.

Largement étudiée au niveau macro-économique, la flexicurité commence seulement à être envisagée au niveau micro. Dans le cadre du projet européen du même nom s'appliquant à trois pays (Belgique, France, Pays-Bas ${ }^{4}$ ), la flexicurité y est définie comme «un compromis dynamique entre besoins d'ajustement de l'activité économique et de l'activité de travail, d'une part, et besoins de minimisation des risques liés à leur interaction, d'autre part» (Pichault \& Xhauflair, 2007, p.9). Elle est ensuite caractérisée par des dimensions polarisées qui permettent de décrire les pratiques observées sur le terrain. Il s'agit donc, pour les entreprises et les salariés, de trouver des compromis à la fois convenables et acceptables, ce qui conduit les auteurs à tendre vers une «normativité construite avec les acteurs, sur la base de réalités et non de postulats idéologiques» (Pichault \& Xhauflair, 2009, p.8). Les deux types d'acteurs directs, employeurs et salariés, sont donc acteurs à la fois en amont (lors de l'élaboration du compromis) et en aval (lors de la mise en œuvre effective de ce compromis) pour permettre une « opérationnalisation » de la flexicurité.

\footnotetext{
${ }^{4}$ www.flexicurity.org
} 
Notre recherche prend donc appui sur ces travaux et souhaite aller plus loin, en identifiant des pratiques de flexicurité, au sens large, à l'œuvre dans des entreprises et en cherchant à comprendre comment ces pratiques parviennent à émerger. Nous retiendrons donc, dans le présent article, une définition large de la flexicurité, entendu comme des dispositifs qui permettent simultanément à l'employeur de gagner en flexibilité et au salarié de gagner en sécurité, dans une logique « gagnant-gagnant».

\subsection{Un nouveau cadre : le niveau méso-organisationnel}

En faisant le choix de centrer notre recherche sur les petites et moyennes organisations (PMO), nous souhaitons à la fois nous intéresser à la très grande majorité des entreprises françaises $(99 \%)$ et à plus de la moitié des salariés français. De même, nous ciblons une population pour laquelle les questions de travail et d'emploi, et plus largement de stratégie des ressources humaines, sont généralement moins prioritaires, la fonction RH dans les PME se caractérisant généralement par sa «présence minimale » (Marchesnay \& Fourcade, 1997).

D'ailleurs, la flexicurité fait déjà l'objet de réflexion sur la prise en compte d'autres niveaux, sectoriels ou territoriaux (Beaucourt, 2008). Le territoire fait partie de l'un des cinq espaces de stabilité professionnelle proposés par Auer et Gazier (2006) : «c'est la proximité, la disponibilité et l'adaptabilité ainsi que la connaissance intime des particularités locales qui permettent à des personnes aux qualifications très diverses de trouver une sécurité de l'emploi. » (p.113). Dans une optique de sécurisation des trajectoires professionnelles, la « gestion territorialisée des trajectoires, grâce à une coordination forte des acteurs présents sur le territoire (entreprises, collectivités locales, service public de l'emploi, partenaires sociaux) » prend toute sa place (Méda \& Minault, 2005, p.34).

Or, si elle apparaît séduisante, la notion de territoire n'en reste pas moins très floue, tour à tour «exténuée » par les approches de développement local de toutes sortes, puis «sublimée » (Pecqueur, 2009), portée notamment par les travaux de l'économie de proximité, qui considèrent que c'est de la rencontre entre une proximité géographique et une proximité organisée qu'émerge le territoire (Zimmermann, 2008). Dans l'approche « proximiste » (Bouba-Olga \& Zimmermann, 2004), l'accent est mis sur le rôle des relations interpersonnelles pour la construction de coopération inter-organisations, tout en s'appuyant sur la proximité spatiale de ces organisations, en réservant «le terme d'espace pour évoquer une proximité physique, le terme de réseau pour évoquer une proximité organisée et, enfin, le terme de territoire pour évoquer la conjonction de ces deux formes de proximité » (p.94).

En s'intéressant à la flexicurité appliquée aux PMO, l'intérêt de raisonner au-delà des frontières traditionnelles de l'entreprise s'accroît encore. En effet, si l'on considère que le modèle fordiste du «château » a laissé sa place à celui du « réseau » (Butera, 1991), les entreprises, et notamment les plus petites d'entre elles, n'ont aujourd'hui d'autre choix que de "coopérer ou périr» (Fourcade, 2008), dans une logique de coopétition (Brandenburger \& Nalebuff, 1997) et d'approche "réticulaire » (Messeghem \& Paradas, 2008). Dès lors, il apparaît que le seul niveau microéconomique ne suffit pas pour comprendre la flexicurité. En effet, d'une part, l'étude d'une seule entreprise ou même de plusieurs entreprises considérées isolément, réduirait l'objet même de la recherche : quelle flexicurité une entreprise peut-elle réellement mettre en place à son seul niveau ? De plus, puisque nous avons fait le 
choix de nous intéresser aux petites structures, la restriction serait encore plus forte : de quels moyens une petite structure peut-elle disposer, alors même que la gestion des carrières «classiques » pose souvent problème ?

Les frontières de l'entreprise ne sont plus constituées de son seul périmètre juridique ou géographique. En Europe, comme dans le monde, des exemples montrent comment des entreprises ont su coopérer et nouer des partenariats pour se développer. Notre analyse de la flexicurité souhaite donc appréhender le niveau « méso », entendu comme l'articulation entre l'industrie, au sens marshallien du terme, et l'espace (Gilly \& Lung, 2005), en considérant ainsi les PMO à un niveau agrégé (Pecqueur, 2009), sur le mode du réseau (Veltz, 2005).

Nous formulons donc l'hypothèse que le niveau « méso » est le plus pertinent pour permettre la mise en œuvre de pratiques de flexicurité (entendue comme davantage de flexibilité et/ou de sécurité pour les employeurs comme pour les salariés) dans les PMO.

\section{Méthodologie et terrains d'investigation}

\subsection{Cadrage méthodologique}

La recherche est à la fois exploratoire et explicative. Exploratoire puisqu'il s'agit de repérer puis d'analyser pour les comprendre les dispositifs mis en œuvre (Girin, 1990). Explicative puisqu'on s'attachera à identifier les facteurs de réussite des cas observés, permettant ainsi de tenter une modélisation qualitative des facteurs clés de succès. Cette recherche recourt donc à l'étude de cas, particulièrement appropriée ici (Eisenhardt, 1989), en l'occurrence multi-sites, et ce pour plusieurs raisons (HladyRispal, 2002). (1) La variété ainsi obtenue permet de valider notre positionnement épistémologique de compréhension de l'objet étudié, tout en cherchant à identifier des savoirs actionnables (Avenier, 2004). (2) L'équilibre recherché dans le choix des cas étudiés permet de faire de cette variété une force de notre travail. En effet, trouver des terrains d'étude ne pose pas vraiment de problème en soi. Par contre, il est plus ardu que ceux-ci soient pertinents par rapport à la problématique traitée et complémentaires les uns des autres. D'où un arbitrage fait entre les différents cas retenus parmi tous ceux susceptibles d'être traités. (3) Le potentiel de découverte de chaque cas est indispensable, afin de collecter le maximum de données, en s'appuyant sur une investigation approfondie auprès des acteurs.

Neuf entretiens semi-directifs ont été menés à titre exploratoire, afin d'identifier les terrains d'investigation. La phase principale est constituée de l'étude de quatre terrains, deux étant des analyses in vivo, ayant fait l'objet d'entretiens semi-directifs, (douze pour le terrain $\mathrm{n}^{\circ} 1$, six pour le terrain $\mathrm{n}^{\circ} 2$ ), conduits sur la base d'un guide d'entretien, retranscrits puis analysés de façon thématique, à la fois verticalement et horizontalement (Gavard-Perret \& Helme-Guizon, 2008). Une triangulation des données est assurée par le recours à l'analyse de données secondaires et à un questionnaire confirmatoire. Les deux autres terrains ont fait l'objet d'une analyse ex post, les expérimentations ayant déjà été menées depuis quelques années lorsqu'ils ont été étudiés. Les données documentaires collectées ont été complétées par des entretiens confirmatoires (deux pour chaque terrain analysé ex post). 


\subsection{Présentation résumée des terrains}

Sur la base des premières informations collectées lors d'une dizaine d'entretiens exploratoires, plusieurs terrains particulièrement pertinents ont été identifiés en fonction des critères suivants : les employeurs doivent être des PMO ; les secteurs d'activité doivent être suffisamment différents pour faire l'objet d'une comparaison stimulante ; la dimension territoriale retenue est celle de la proximité géographique relative des employeurs entre eux, le département constituant l'échelon maximal ; des pratiques de flexicurité, ou des tentatives, doivent effectivement être en œuvre dans les structures étudiées, au sens où l'employeur comme le salarié y gagnent, conformément à la définition large que nous avons déjà donnée de la flexicurité. Quatre terrains ont finalement été étudiés :

- Cas $n^{\circ} 1$. Le secteur du sport en Auvergne, en présentant la particularité de concerner principalement des associations, constitue un vrai champ d'investigation, compte tenu du contexte institutionnel et législatif et surtout des spécificités sociales rencontrées (précarité de la main-d'œuvre, employeurs de très petite taille, notamment). La récente signature d'un contrat d'objectifs régional fait de la mutualisation des compétences un axe majeur, en s'appuyant notamment sur quatre groupements d'employeurs (GE) qualifiés d'exemplaires.

- Cas $n^{\circ}$ 2. Le Parc Naturopôle Nutrition Santé, créé autour d'une première entreprise installée en milieu rural par la seule volonté de son dirigeant, est une zone d'activité thématique qui regroupe aujourd'hui quatre PME, alliant vision stratégique commune et souci d'une gestion « humaine » de leurs salariés. En matière de flexicurité, plusieurs actions concrètes existent : de la mobilité professionnelle à la formation mutualisée, en passant par la mise à disposition de personnel.

- Cas $n^{\circ} 3$ : le territoire de Saint-Flour Haute-Auvergne qui a fait l'objet de deux études au cours des dix dernières années, afin de permettre à des travailleurs saisonniers de compléter leur activité. Malgré l'implication des acteurs directs (entreprises, salariés) et indirects (chambres consulaires, SPE...) lors de ces études, et malgré des préconisations intéressantes et réalistes, aucune action n'a été mise en place.

- Cas n²4. En 2005, une étude a été menée sur la mutualisation des ressources humaines sur le territoire de GAZACA, association regroupant les zones d'activités de l'agglomération de Clermont-Ferrand (Bernon et al., 2006). L'étude a notamment préconisé la création d'un bureau des mobilités, dont la mise en œuvre a été très rapidement abandonnée.

\subsection{Les contours de la flexicurité rencontrée}

\subsubsection{Les GE du Sport en Auvergne}

Le Contrat d'Objectifs régional pour le développement des métiers et de la formation professionnelle dans le Sport, signé en novembre 2008, fait de la mutualisation des compétences un axe majeur. L'idée consiste à s'appuyer sur des exemples réussis en matière de mise à disposition de professionnels, à permettre aux employés du secteur de cumuler le statut de salarié et celui d'indépendant et à inciter au développement de la «plurivalence », autrement dit favoriser le développement de compétences transversales et/ou la bi-qualification. Quatre Groupements d'Employeurs (GE) sont explicitement reconnus par ce contrat d'objectifs et font l'objet d'un premier champ d'investigation (tableau 1, page suivante). 
Tableau 1 : Caractéristiques des quatre GE étudiés

\begin{tabular}{|l|l|c|l|}
\hline & $\begin{array}{l}\text { - Date création } \\
\text { > Origine }\end{array}$ & $\begin{array}{c}\text { Nombre } \\
\text { adhérents }\end{array}$ & \multicolumn{1}{|c|}{ Effectif } \\
\hline GE2A & $\begin{array}{l}\text { - Juin 2008 } \\
\text { > ex-Profession Sport Allier }\end{array}$ & 180 & $\begin{array}{l}90 \text { salariés } \\
\text { (sport + socioculturel })\end{array}$ \\
\hline GE Sports 63 & $\begin{array}{l}\text { - Juin 2007 } \\
\text { > Comité Départemental } \\
\text { de Tennis de table }\end{array}$ & 23 & $\begin{array}{l}8 \text { salariés (=3 ETP) dont } \\
1 \text { responsable sportif } \\
\text { (CDI temps plein) } \\
\text { 7 entraîneurs itinérants } \\
\text { CDI dont 6 intermittents } \\
\text { et 1 temps plein. }\end{array}$ \\
\hline $\begin{array}{l}\text { GE Sport } \\
\text { Auvergne }\end{array}$ & $\begin{array}{l}\text { - Septembre 2008 } \\
\text { > CROS }\end{array}$ & 12 & $\begin{array}{l}2 \text { salariées } \\
\text { dont 1 temps plein }\end{array}$ \\
\hline GEFSA & $\begin{array}{l}\text { - Février 2009 } \\
\text { > Ligue d'Auvergne de Football }\end{array}$ & N.S. & $\begin{array}{l}3 \text { salariés } \\
\text { dont 2 temps plein }\end{array}$ \\
\hline
\end{tabular}

L'idée directrice de ces GE est de concilier les besoins des adhérents, principalement constitués de petites associations sportives ayant un faible rayonnement géographique, pour lesquels il est primordial de disposer d'encadrants professionnels dûment qualifiés pour un nombre d'heures souvent restreint, avec les attentes des salariés, qui souhaitent pouvoir vivre de leur métier, notamment en effectuant un nombre suffisant d'heures hebdomadaires. «Aujourd'hui, les demandes [d'encadrants dans les clubs], c'est beaucoup du 18h-20h et on ne fait pas un temps plein en bossant tous les jours de $18 \mathrm{~h}$ à 20h!» (responsable sportif du GE Sports 63) ${ }^{5}$. Cette difficulté oblige les encadrants à se tourner vers le multisalariat, le GE apportant une facilité de prospection, en permettant simultanément d'élargir les créneaux horaires, les publics et les disciplines enseignées : «Le souci, souvent, c'est que les heures de sport sont toutes les mêmes, donc on ne peut pas faire du temps plein avec ça. Quand on se base sur tous les clubs, on a des publics différents (école, seniors en journée...), grâce au territoire. Et c'est plus facile d'être sur un territoire, les gens se connaissent. » (chargée de mission du GE Sport Auvergne). La typologie des employeurs est également élargie : «L'idée [de créer un GE] était donc de voir, à partir de l'identification d'une structure de football qui prendrait un emploi pour $10 \mathrm{~h}$ par semaine, comment l'aider à compléter ce temps partiel en apportant d'autres structures associatives football, ou sportives, ou socioculturelles, tout ce qui tourne autour de l'éducation, les communes, voire l'emploi privé. » (Directeur Technique Régional de la Ligue de Football d'Auvergne).

Globalement, les GE du sport en Auvergne contribuent effectivement à une logique de « gagnant-gagnant », à trois niveaux : pour les structures adhérentes, qui professionnalisent leur encadrement mais aussi, et surtout, simplifient leurs obligations administratives et sécurisent leurs relations d'emploi ; pour les salariés, qui, par l'intermédiaire

\footnotetext{
${ }^{5}$ Tous les entretiens des GE du Sport en Auvergne ont été menés en février 2009.
} 
du GE, structurent leur emploi, en augmentant le nombre d'heures travaillées et en tirant ainsi un revenu décent, en simplifiant la gestion de leur multisalariat, prise en charge par le GE et en bénéficiant de formations développant la plurivalence (en se formant à une autre discipline ou en s'ouvrant à d'autres publics) ; mais aussi pour le territoire, puisque la proximité géographique entre les différentes structures d'accueil de chaque salarié s'avère indispensable, pour des raisons évidentes de temps et de coûts de déplacement, mais aussi parce que les acteurs territoriaux tirent des bénéfices directs (en tant qu'adhérents, par exemple pour l'encadrement scolaire) et indirects (puisque les GE permettent de soutenir l'activité du territoire) de ces GE : «Il y a une demande de la part d'un club (10h) et donc je recherche des compléments d'heures, afin d'avoir une activité complète, dans un périmètre géographique assez restreint pour que la personne n'ait pas trop de déplacements. [...] J'ai contacté la Communauté de Communes. Les gens sont séduits par le projet: c'est quelqu'un qui restera sur la commune, qui sera sédentarisé, ça amène de la vie. » (Directeur du GEFSA).

\subsubsection{Le Naturopôle}

Situé sur la petite commune de Saint-Bonnet-de-Rochefort dans l'Allier, à une trentaine de kilomètres de Vichy, le Naturopôle accueille quatre entreprises du secteur de l'aliment santé, organisées en micro-cluster, c'est-à-dire capables d'apporter une « solution globale » à leurs clients, grâce à une complémentarité entre l'étape du processus de production à laquelle elles interviennent, le marché auquel elles s'adressent et la spécialité préparée. Elles accueillent un effectif total d'environ 170 salariés, à rapporter à la population locale de 650 habitants. Le Naturopôle est par ailleurs labellisé PER (Pôle d'Excellence Rurale) ${ }^{6}$.

L'ambition de l'initiateur du Naturopôle, était d'entreprendre à la campagne. Son objectif était de prouver que l'on peut créer une entreprise en milieu rural, en créant de l'emploi pour maintenir et développer des bassins de vie. L'idée était également de démontrer que l'on peut avoir des services et des activités pointues, requérant une certaine expertise technique et technologique, sans avoir nécessairement besoin de s'implanter dans une grande ville. Pour la Responsable Ressources Humaines : «Créer de l'emploi à la campagne, c'est une vraie conviction. Ce n'est pas pour répondre à un effet de mode. $\gg^{7}$.

Des expérimentations sont menées depuis quelques années entre les entreprises du Naturopôle, notamment en matière de mutualisation des ressources humaines. Ainsi la mobilité professionnelle est-elle rendue possible d'une entreprise à l'autre (promotion, changement de poste, mais aussi reclassement suite à un accident du travail ou des contraintes médicales). De même, le prêt de personnel peut être pratiqué occasionnellement. La formation fait l'objet de certaines actions collectives, surtout dans des domaines transversaux comme le management. Enfin, la plus grosse entreprise du site, LPH, adhère depuis peu à un GE, avec un intérêt bien compris : "LPH est adhérent du GE et s'engage pour une période tous les ans. C'est moins souple que l'intérim pur. Mais ça per-

\footnotetext{
${ }^{6}$ Les PER (Pôles d'Excellence Rurale) sont issus d'un appel à projets lancé par la DIACT (Délégation Interministérielle à l'Aménagement et à la Compétitivité des Territoires) en décembre 2005 dans le but de soutenir « des initiatives locales porteuses de projets créateurs d'emplois, innovants, ambitieux, bâtis autour de partenariats publics-privés. » 379 projets ont été labellisés en 2006, et 115 en 2010.

${ }^{7}$ Les entretiens du Naturopôle ont été menés entre avril et juillet 2009.
} 
met d'avoir des personnes qui reviennent tous les ans, qui connaissent leur métier. » (Directrice du GE Activa). Enfin, la place de la R\&D étant déterminante dans un secteur aussi concurrentiel que celui des alicaments, les entreprises du Naturopôle se doivent d'innover dans leurs domaines de savoir-faire (notamment les pro-biotiques et les antioxydants). Mais l'innovation coûte cher, et elle est limitée par l'autofinancement, qui était jusqu'alors le principe dominant, assumé par les dirigeants des entreprises du Naturopôle. Aujourd'hui, celles-ci nouent des partenariats permettant de financer une partie de la R\&D. Sur deux innovations éminemment stratégiques pour le Naturopôle (et de ce fait confidentielles), un système de mutualisation de la main-d'œuvre hautement qualifiée a été mis en place, sous forme de grands projets collaboratifs en cours de constitution.

Si le terme de «flexicurité » n'a jamais été utilisé spontanément par les personnes rencontrées au sein du Naturopôle, les différentes actions qui viennent d'être présentées s'en rapprochent. D'ailleurs, sollicitée sur ce terme, la RRH précise : «On n'a pas attendu de parler de flexicurité pour en faire. Pour moi, ça renvoie à plein de choses : la formation, la politique RH au sens large. On attache beaucoup d'importance à l'Homme, donc c'est important de faire évoluer les personnes dans leur métier. » Le cas du Naturopôle nous semble particulièrement riche dans le cadre de notre recherche, avec une démarche de mutualisation, certes timide mais néanmoins efficace, qui cherche bien à concilier l'intérêt des entreprises, qui ont besoin de compétences définies à des périodes données et celui des salariés, qui, grâce à ces actions, bénéficient de perspectives plus intéressantes qu'en raisonnant au sein d'une seule entreprise.

\subsubsection{La pluriactivité sur le territoire de Saint-Flour Haute-Auvergne}

Le Pays de Saint-Flour Haute-Auvergne est un territoire rural de moyenne montagne, caractérisé par une très faible densité et une population totale de 41.000 habitants. L'économie se construit autour de la valorisation de ses ressources locales : agriculture de montagne ; tourisme d'hiver et d'été. L'agriculture est le secteur économique prépondérant, l'agroalimentaire constituant l'unique secteur industriel présent sur le Pays, avec la filière Bois, en cours de structuration localement. L'artisanat, le commerce et le tourisme représentent les principales « entreprises » du territoire.

Deux études constituent le point d'entrée de notre recherche, traitant d'une problématique commune : pérenniser l'emploi en améliorant les conditions de la saisonnalité. À l'origine de la première étude se situe, dès 1998, la demande du directeur de site de la société exploitante des remontées mécaniques de la station de sport d'hiver du Lioran, dont l'objectif était de pérenniser l'emploi de ses salariés saisonniers. Un diagnostic territorial a mis en évidence la nécessité de transformer l'emploi saisonnier en emploi durable et a identifié trois axes stratégiques : faciliter l'émergence de nouveaux gisements d'emploi en encourageant la pluriactivité dans les secteurs les plus soumis aux variations saisonnières, notamment le tourisme, l'agriculture et l'environnement, et en mettant en place une formation-action à l'attention des saisonniers ; réaliser un « schéma de l'emploi saisonnier » et permettre ainsi de vérifier (ou d'encourager) l'adéquation entre les besoins des entreprises et ceux des saisonniers, notamment en matière de formation ; améliorer la saisonnalité via la création d'une "Maison des saisonniers », une étude de faisabilité ayant été menée auprès de 132 Maires appartenant à la zone territoriale concernée. En juin 2003, le bilan dressé permet d'établir deux constats : une forte mobili- 
sation des membres du Comité de Pilotage (Sous-Préfecture, DDTEFP, Communauté de Communes, AGEFOS-PME) autour des différents axes de travail, d'une part ; la volonté d'apporter une réponse globale à la problématique de la saisonnalité, d'autre part. Dans un premier temps, le projet a d'ailleurs obtenu des résultats satisfaisants : 20 saisonniers, travaillant soit à la station du Lioran, soit aux thermes de Chaudes-Aigues, ont effectivement été formés dans le cadre de la formation-action destinée à promouvoir la pluriactivité ; le schéma de l'emploi saisonnier a effectivement été réalisé, faisant ressortir le besoin d'un accompagnement global des travailleurs saisonniers ; l'opportunité de créer un GE a été étudiée et a fait l'objet d'une estimation fine de sa faisabilité ; le projet le plus emblématique, la Maison des Saisonniers, a fait l'objet d'une étude de faisabilité, avec des résultats tout à fait positifs. Pourtant, quel que soit l'axe de travail considéré parmi les trois cités précédemment, aucun avancement n'a été constaté par la suite.

Malgré les suites décevantes de ce premier projet, la problématique de la saisonnalité restait posée. Ainsi, en novembre 2005, le Préfet du Cantal a lancé une vaste réflexion sur la pluriactivité du Pays de Saint-Flour Haute-Auvergne. L'objectif revendiqué était de « produire un état des lieux de la réalité de la pluriactivité sur le territoire du Pays de Saint-Flour Haute-Auvergne », dans la continuité du projet territorial conduit entre 1999 et 2003. Plus largement, l'étude avait une double finalité : cerner les attentes et les freins des individus et des entreprises du bassin d'emploi au regard de la pluriactivité ; engager localement un débat avec les entreprises et les individus, pour favoriser le développement des pratiques de pluriactivité. La différence la plus sensible avec l'étude précédente tient dans la volonté d'élargir la réflexion à la pluriactivité, tout en partant de la notion de travail saisonnier, qui constitue une composante importante de l'économie locale du territoire considéré. Une étude a donc été menée auprès de trois publics : les entreprises, d'une part, interrogées sur les thématiques des besoins d'emplois, des projets de développement, du degré d'intérêt pour un dispositif de travail à temps partagé, et de la mise à disposition d'un fichier de travailleurs saisonniers ou pluriactifs ; les individus pluriactifs, d'autre part, en analysant le profil des individus, les emplois occupés, les formations suivies et les besoins spécifiques (logement...) ; les exploitants agricoles, enfin, sous forme d'exploitation des données statistiques disponibles, puis d'enquête auprès des exploitants pluriactifs identifiés sur le territoire. Cette étude a pris en compte la pluriactivité sous ses différentes formes, sans la limiter au seul tourisme, et d'identifier le poids déterminant de la pluriactivité agricole, à la fois comme employeur potentiel et comme salariés potentiels. Elle a également contribué à estimer quantitativement le nombre de pluriactifs sur le bassin de Saint-Flour, tout en rendant compte de la diversité des situations, au niveau des entreprises comme des individus et en soulignant le manque d'information global sur les plans juridique et pratique, et plus particulièrement sur l'aspect de la formation.

Les réflexions engagées sur le territoire de Saint-Flour Haute-Auvergne constituent bien la recherche d'une forme de flexicurité. En effet, nous retrouvons ce souci de concilier davantage de sécurisation pour les salariés et de flexibilité pour les entreprises. Ces dernières sont parfois soumises à des aléas, par définition imprévisibles, qui justifient de recourir à une flexibilité numérique externe pour adapter les effectifs au plan de charge. Or ces variations peuvent également être saisonnières, donc éventuellement complémentaires entre l'hiver (Le Lioran) et l'été (activités touristiques sur l'ensemble du département, ou thermales à Chaudes-Aigues, par exemple). Mais celles-ci ne représentent en réalité que la 
moitié des situations de pluriactivité, qui doit donc être étudiée au cas par cas. Globalement, le résultat le plus intéressant semble être la recherche d'une forme de mutualisation du personnel, sous certaines réserves8, l'intérêt majeur consistant à garder les compétences sur le territoire, à disposition des entreprises qui y font régulièrement appel.

Du côté des salariés, la pluriactivité peut être choisie (notamment dans le secteur agricole), mais aussi subie. La dimension de proximité géographique entre le lieu de résidence du salarié et son lieu de travail semble déterminante (et se heurte, dans le cas contraire, à des difficultés de logement). Sur le plan des compétences du salarié, la polyvalence (au sens d'une variété d'activités) peut être perçue comme un avantage pour lui, en termes de facilité à trouver un emploi, alors que le manque de formation est clairement ressenti, à la fois par les employeurs et par les salariés eux-mêmes. De plus, sur le plan temporel, la pluriactivité semble être une forme de flexicurité à l'année, alors qu'elle n'apporte pas de garantie à moyen et long terme.

Ces études sur le territoire de Saint-Flour Haute-Auvergne s'inscrivent donc parfaitement dans le cadre de notre recherche, puisqu'elles traitent de formes particulières d'emploi, qui tentent de mettre en adéquation les besoins et attentes des entreprises et ceux des salariés.

\subsubsection{GAZACA : des zones d'activités à l'épreuve de la mutualisation}

En mai 2002, les six principales zones d'activités de l'agglomération clermontoise se sont regroupées au sein de l'association GAZACA (Groupement des Associations des Zones d'Activités de Clermont Auvergne), regroupant 1.300 entreprises pour quelque 20.000 salariés. En 2005 l'ARACT Auvergne lance un projet pour le développement de la gestion des ressources humaines dans les entreprises de GAZACA, à travers la mutualisation, vue comme une mise en commun des outils et des expériences. L'objectif du projet, tel qu'il est présenté dans le rapport final, est de «montrer en quoi la mutualisation des expériences et des outils pour la gestion du personnel et des ressources humaines sur un territoire peut aider les entreprises à faire face aux difficultés. » Les trois clés d'entrée de l'étude recoupent, partiellement ou en totalité, notre propre problématique : la question des pratiques RH, plus particulièrement dans les TPE-PME, constitue le socle de l'étude, l'originalité se situant dans la confrontation du point de vue des dirigeants avec celui des salariés, conformément à la vocation même de l'ARACT, instance paritaire ; le territoire fait l'objet d'une hypothèse qui sous-tend la suite des travaux, celle que «sur un territoire centré sur l'économique, [...]la mutualisation pouvait être une action déterminante pour favoriser l'émergence de territoires originaux, capables de stimuler la vie sociale de la zone au bénéfice de ses habitants : à la fois des entreprises et de leurs salariés. »; la mutualisation est définie comme la mise en commun, c'est-à-dire "partager des ressources, disposer de forces que l'on met à disposition des autres qui rétribuent en contrepartie, créer des forces partagées pour lesquelles chacun assumera une partie et profitera du tout. ». Si le terme de flexicurité n'est pas utilisé, l'optique de l'étude qui est d'améliorer les conditions de travail, au profit des dirigeants comme des salariés, est effectivement concordante avec le concept tel que nous l'avons préalablement défini.

\footnotetext{
${ }^{8}$ Selon le rapport (p.69) : « la concurrence au cœur du secteur de l'hôtellerie restauration bloque l'emploi à temps partagé. »
} 
La première phase d'étude et d'enquête consistait en des monographies d'entreprises centrées sur les pratiques en ressources humaines puis une enquête par questionnaire auprès des employeurs et des salariés. Une typologie des entreprises en matière de RH a alors été proposée (Bernon et al., 2006), et des préconisations ont été formulées, notamment celle d'un bureau des mobilités, destiné à «faciliter le recrutement, la mobilité et la recherche d'emplois sur les zones d'activités [et de permettre] le croisement des difficultés de recrutement des dirigeants et des besoins de changement des salariés. » L'idée s'appuie sur un double constat : du côté des dirigeants, il s'agit de remédier aux difficultés de recrutement par rapport à des compétences bien identifiées ; du côté des salariés, il s'agit d'offrir des réponses aux besoins de reconnaissance, d'évolution professionnelle et de perspectives de carrières. L'outil proposé visait à mettre en place un «bureau des mobilités » permettant de «visualiser les compétences demandées sur un territoire et non plus uniquement les offres d'emploi », en partant du constat fait dans le questionnaire que le salarié est prêt à changer d'entreprise, mais pas de territoire. Pour cela, un partenariat avec l'ANPE a été créé sous la forme d'un contrat de service, pour mettre en place un service de proximité, consistant en une permanence, ouverte à titre de test sur l'une des ZA, à raison d'une demi-journée par quinzaine. L'information a été assurée auprès des entreprises par le biais d'une plaquette spécifique et par le site Internet de GAZACA, une plaquette ayant été réalisée conjointement par GAZACA et l'ANPE à destination des salariés. Or, après quelques semaines de fonctionnement seulement, ce bureau des mobilités a cessé de fonctionner. "L'idée du bureau des mobilités était très délicate à porter. [...] Pour les entreprises, le risque était de les «vider » de leurs compétences au profit de la concurrence. » On peut aussi, à l'instar de certains acteurs, s'interroger sur la notion même de territoire au sein d'une ZA : «Il faut vraiment une solidarité pour constituer un territoire cohérent. Dans une ZA, en fait, on aligne des concurrents. »

\section{La flexicurité dans les PMO : une alchimie entre innovation sociale et territoire}

\subsection{Une flexicurité réelle mais non aboutie : une flexicurité "émergente »}

\subsubsection{Attributs de la flexicurité observée}

Les quatre cas étudiés ont mis en pratique, ou tenté de le faire, des formes de flexicurité, au sens de la définition large que nous en avons donnée, axée autour de davantage de flexibilité et de sécurité.

Tout d'abord, la flexicurité constatée (ou recherchée) dans les quatre cas est réelle. En toute logique, et conformément à ce qui semble caractériser le plus la flexicurité, la recherche d'une plus grande flexibilité pour l'employeur est présente. Pour les salariés, la sécurisation est également à l'œuvre. Dans tous les cas, il s'agit de structurer l'emploi, en permettant d'avoir une visibilité de plusieurs mois sous forme d'un complément d'activité (GE du Sport, Saint-Flour Haute-Auvergne) ou de permettre des transitions professionnelles facilitées (GAZACA). Ces éléments n'apportent rien de nouveau quant à une meilleure connaissance de la flexicurité sous l'angle pratique. Ils ne font finalement que confirmer ce que l'on est en droit d'attendre de la flexicurité, au niveau micro, compte tenu des définitions et différents travaux déjà menés. 
De façon peut-être moins évidente, notre recherche montre que la flexicurité ne se résume pas à la vision caricaturale à laquelle on s'arrête trop souvent, à savoir de la flexibilité pour l'employeur et de la sécurité pour le salarié. Il apparaît clairement que l'employeur souhaite également sécuriser sa relation d'emploi. Cela se traduit par exemple dans le cas des GE du Sport par les raisons mêmes d'adhésion au GE, en vue de simplifier et sécuriser la relation juridique et les formalités administratives liées à l'embauche et à la gestion de personnel. De même, dans le cas du Naturopôle, l'entreprise bénéficie de compétences reconnues, dans des conditions juridiquement et financièrement favorables (pas de licenciement à prévoir, embauche au prorata des besoins réels). Enfin, dans le cas de Saint-Flour, les employeurs souhaitent également simplifier et clarifier le recours à de la main-d'œuvre plurivalente, en fidélisant le personnel compétent et en rentabilisant les investissements de formation. Les salariés s'inscrivent aussi, parfois, dans une recherche de flexibilité. Dans le cas des GE du Sport, nous avons identifié certains salariés qui ne souhaitent pas travailler plus, pour des raisons variées. Le fonctionnement des entreprises du Naturopôle permet à certains salariés d'avoir des opportunités de carrière, dans le sens de "trajectoires obliques » (Auer \& Gazier, 2006). Dans le cas de Saint-Flour Haute-Auvergne, également, les salariés « pluriactifs choisis » précisent vouloir garder leur liberté, donc une certaine forme de flexibilité. Dans le cas de GAZACA, enfin, les salariés aspirent à pouvoir évoluer professionnellement, quitte à changer d'employeur.

\subsubsection{Une flexicurité « imparfaite»}

Si les éléments qui viennent d'être évoqués permettent de confirmer que les pratiques étudiées représentent bien des formes de flexicurité, il convient néanmoins de mettre l'accent sur deux limites, fortes, rencontrées : celles liées à l'horizon temporel dans lequel s'inscrivent les pratiques de flexicurité ; celles concernant la notion même de plurivalence, et plus largement, ce que l'on attend de la flexicurité.

Tout d'abord, la question de la pérennité même des dispositifs mis en œuvre apparaît essentielle. Dans le cas du secteur du sport, comment les GE, très récents pour trois d'entre eux, vont-ils assurer l'assise financière nécessaire ? Certains se sont déjà emparés du problème et s'attachent à calculer des coûts de revient très précis. La question du coût des prestations du GE est donc primordiale et renvoie directement aux moyens financiers dont disposent les associations adhérentes. Si les GE ne bénéficient que de faibles subventions directes ${ }^{9}$, les associations utilisatrices ne peuvent en revanche adhérer et utiliser le GE que grâce aux subventions qu'elles sollicitent au niveau de leur territoire notamment (Mairies, principalement). De plus, à l'instar de ce que faisaient les associations, les GE utilisent abondamment les contrats aidés aujourd'hui en vigueur, en apportant d'ailleurs sur ce sujet un vrai rôle de conseil et d'expertise ${ }^{10}$, qui sert aussi bien aux adhérents qu'aux salariés. Cependant, un contrat aidé ne l'est qu'un temps, et le salarié qui ne remplit plus les conditions d'éligibilité ne risque-t-il pas de retomber dans la précarité, au prétexte d'un coût trop élevé pour le GE comme pour les adhérents ? De même, la facturation des déplacements grève vite le budget des adhérents installés en milieu rural.

\footnotetext{
${ }^{9}$ Aide à la création de GE du Conseil Régional d'Auvergne.

${ }^{10}$ Par exemple, le GE2A fonctionne en liaison étroite avec le dispositif Impact Emploi de l'URSSAF.
} 
Certains départements prennent donc en charge une partie des coûts pour ne pas pénaliser ces clubs, voire incitent à l'utilisation de main-d'œuvre mutualisée ${ }^{11}$. Il s'agit donc d'un système sous perfusion de fonds publics, à plus ou moins grande échelle.

D'autre part, et toujours concernant l'horizon temporel, les mécanismes de flexicurité mis en œuvre ne s'inscrivent pas dans le long terme, contrairement à ce que préconisait le contrat d'activité (Boissonnat, 1995) ou les droits de tirage sociaux (Supiot, 1999). On ne raisonne pas, ici, à l'échelle d'une vie, mais plutôt à l'échelle d'une ou, au mieux, de quelques années. Les deux exemples les plus révélateurs sont ceux de Saint-Flour Haute-Auvergne, dans lequel on cherche à compléter l'activité, souvent saisonnière au départ, et celui des GE du sport, où l'on reconstitue un temps plein à partir de « morceaux d'emploi », mais sur une saison seulement.

Ceci nous conduit à un premier résultat issu de notre recherche : si les dispositifs étudiés s'avèrent bien être des formes de flexicurité, ils n'en sont en revanche que des esquisses, tentatives imparfaites et incomplètes. Cet horizon temporel restreint mérite d'ailleurs d'être examiné au regard de la littérature. En effet, le concept de PME, qui fait l'objet depuis plusieurs années de multiples recherches, est souvent qualifié, entre autres, par le court-termisme dont font preuve les dirigeants. Ainsi, le dirigeant de PME est sensible à la proximité temporelle, à savoir l'intérêt pour ce qui est immédiat, et une difficulté, voire une incapacité, à se projeter dans l'avenir (Torrès, 2007). On pourrait alors formuler l'hypothèse que le court-termisme de la flexicurité observée dans les petites structures étudiées serait davantage imputable à cette proximité temporelle caractéristique des PME-PMO, qu'à un déficit qualitatif ou quantitatif de la flexicurité en elle-même.

La seconde limite concerne la plurivalence, évoquée à plusieurs reprises dans les terrains étudiés. Dans le cas des GE du Sport, elle est affirmée comme un élément clé du contrat d'objectifs. Plus largement, quel que soit le cas étudié, la formation apparaît comme incontournable pour rendre le salarié plus «employable », et, par conséquent, mieux outillé pour trouver en emploi, compléter le sien, ou en trouver un nouveau. "Initialement, l'emploi était le référent stable et l'employabilité n'était rien d'autre qu'une collection d'aptitudes ou des comportements permettant d'obtenir et de conserver cette position stable dont l'idéal était la relation salariale à long terme, voire l'emploi à vie. » (Gazier, 2006). Or il semble qu'on assiste aujourd'hui à une inversion de la relation entre emploi et employabilité et la place qui est maintenant donnée à cette dernière. Ainsi, par exemple dans le cas des GE du sport, les salariés sont fortement incités à se former par l'intermédiaire du GE et en vue de permettre à ce dernier de compléter plus facilement les horaires de travail. En même temps, ces salariés développent des compétences propres, qui leur seront utiles, plus tard, même s'ils quittent le GE. On est donc bien, ici, en présence d'une forme d'employabilité demandant au salarié un effort, personnel et professionnel, en vue de se prendre en charge, pour et à travers le GE qui l'emploie au moment considéré, mais aussi dans une perspective de carrière plus autonome. De même, dans le cas de l'adhésion de LPH, entreprise du Naturopôle au GE Activa, le salarié est acteur de son parcours en acceptant de travailler dans deux entreprises très différentes (les Thermes de Vichy et LPH), ce qui, certes, nécessite de sa part un effort d'adaptation et de formation, mais lui apporte en retour une activité tout au long de l'année.

\footnotetext{
${ }^{11}$ Le Conseil Général de l'Allier finance ainsi $50 \%$ des frais de déplacement en milieu rural.
} 
La notion d'employabilité renvoie, assez logiquement, à celle de polyvalence, autour de laquelle règne un malentendu, puisqu'elle recouvre en réalité deux phénomènes bien distincts (Everaere, 2008) : la polyvalence par nomadisme, généralement mal perçue par les salariés, qui se traduit par la rotation sur des postes de travail distincts, et contribue ainsi à disperser et donc affaiblir les compétences ; la polyvalence « noble », par recomposition des tâches, qui permet d'élargir et d'enrichir les compétences, grâce à la variété des tâches confiées, et à l'autonomie dont jouit le salarié, mais nécessite une certaine sédentarisation pour permettre la maîtrise progressive de cette variété qualitative et quantitative du travail. Une telle distinction se rencontre dans les cas étudiés, avec la flexicurité comprise comme une façon, pour le salarié, de rester maître de ses choix. Ainsi dans le cas de Saint-Flour Haute-Auvergne, la pluriactivité choisie se rapproche de la polyvalence «noble » alors que la pluriactivité subie relève plutôt d'une polyvalence par nomadisme. Cette approche nous paraît devoir être complétée par celle de « compétence » comprise comme «la prise d'initiative et de responsabilité de l'individu sur des situations professionnelles auxquelles il est confronté » (Zarifian, 1999). Au niveau individuel, il s'agit d' "une combinaison de multiples ressources, liées à l'expérience ou à la formation de la personne, mais aussi à la situation de travail dans laquelle elle se trouve, qui rend une personne "capable de..." dans un contexte précis » (Defélix et al., 2006). Plus largement, il nous semble intéressant d'évoquer à ce propos une forme plus récente de la division du travail, celle du réseau. Nous avons déjà évoqué «l'entreprise réticulaire ». Il s'agit maintenant de faire le lien avec la flexibilité que donnent les possibilités de «déconstruction / reconstruction du réseau en fonction des opportunités, d'autant plus aisées que les éléments de base peuvent être membres de plusieurs réseaux et qu'ils peuvent facilement transférer leur activité (ou leur motivation s'agissant d'individu) d'un réseau à un autre. » (Igalens, 2006, p.296). En prenant en considération cette dimension, la flexicurité telle qu'étudiée semble être un outil au service du développement des compétences individuelles, qui fonctionne d'autant mieux que les individus font eux-mêmes partie de réseaux, à l'intérieur desquels ils peuvent proposer (et donc mobiliser) leurs compétences.

Les pratiques observées apparaissent bien comme des formes de flexicurité. Cependant, celles-ci semblent tout à fait incomplètes au regard des ambitions premières de la flexicurité, notamment en matière d'horizon temporel. Certains considéreront que nous sommes davantage en présence de dispositifs de «non-précarité », qui évitent simplement à une certaine catégorie de salariés de se retrouver en marge du marché du travail. Nous rejoignons ici les courants de pensée sur le dualisme du marché du travail, opposant un «marché primaire », avec des salariés stabilisés, pour lesquels la flexicurité n'est pas d'actualité ou qui organisent eux-mêmes leurs trajectoires, dans le cadre, par exemple de la polyvalence noble, et un «marché secondaire », avec des salariés généralement peu qualifiés et sans perspective de carrière, qui subissent une polyvalence nomade, et pour lesquels la flexicurité se traduit d'abord par une recherche de stabilisation, au moins à court terme, de leurs trajectoires. Il nous semble pourtant que les démarches étudiées s'inscrivent bien dans ce que la flexicurité, au sens plein du terme, devrait être, même si elles ne font que tendre vers celle-ci. Les rudiments de ce que doit être la flexicurité sont en place, avec la tentative de conciliation des attentes et besoins des employeurs comme des salariés. Nous proposons donc d'utiliser le terme de «flexicurité émergente » dans la suite de notre discussion pour désigner les formes 
observées et pour bien spécifier que nous considérons avoir observé des dispositifs somme toute imparfaits et incomplets au regard de la littérature.

\subsection{Le territoire comme point d'ancrage}

Selon les cas observés, le territoire considéré est plutôt rural (Naturopôle, Saint-Flour), plutôt urbain (GAZACA) ou mixte (GE du Sport). Cette dimension, qui fait partie intégrante du contexte de chaque cas, ne semble pas avoir d'impact majeur sur le degré de réussite des dispositifs de flexicurité mis en œuvre (tableau 2).

Tableau 2 : La variable « rural / urbain »

\begin{tabular}{|l|c|c|}
\hline \multicolumn{1}{|c|}{ Cas } & expérience & milieu \\
\hline 1. GE du Sport & réussite & Mixte (rural et urbain) \\
\hline 2. Naturopôle & réussite & Rural \\
\hline 3. Saint-Flour & échec & Rural \\
\hline 4. GAZACA & échec & Urbain \\
\hline
\end{tabular}

En revanche, la notion de proximité semble, elle, déterminante, et peut s'entendre selon les deux modalités de l'économie de proximité (Bouba-Olga \& Grossetti, 2008) : spatiale d'une part, caractérisée par une faible distance entre les différents acteurs : il s'agit du périmètre géographique relativement restreint au sein duquel s'organise le réseau d'employeurs ; organisée, d'autre part, caractérisée alors par le niveau de coopération entre ces acteurs, en amont du projet de flexicurité : en d'autres termes, un réseau, formalisé ou non, existait-il entre les employeurs avant les projets de flexicurité étudiés.

Ainsi le territoire joue un rôle majeur, dans le sens premier d'une proximité géographique. Pourtant le simple fait d'être installé à côté de quelqu'un n'induit pas automatiquement coopération, comme le montrent les relations de voisinage qui peuvent être quasi-absentes, voire désastreuses, pour des raisons aussi variées que la rareté de certaines ressources ou des expériences précédentes malheureuses (Rallet \& Torre, 2004). Dans le premier cas, la rareté des ressources renvoie clairement, parmi les cas étudiés, à la frilosité des entreprises de GAZACA face au bureau des mobilités, comme le formulent tour à tour deux acteurs de ce projet : «Les entreprises sont toujours méfiantes quant à la mutualisation de leur personnel. Il y a toujours une concurrence latente et la peur de se faire piquer leurs bons collaborateurs. » (Secrétaire Général de la CGPME Auvergne) ${ }^{12}$; "Pour les entreprises, le risque [du bureau des mobilités] était de les "vider» de leurs compétences au profit de la concurrence. » (Trésorier de GAZACA). Dans le second cas, l'historique qui précède l'action considérée peut jouer en la défaveur de la coordination entre acteurs. Dans les cas étudiés, c'est encore GAZACA qui illustre le mieux cette situation, avec des acteurs périphériques qui ont des attentes divergentes de l'action menée, mais aussi un passé générateur de tensions dans leurs relations inter-organisationnelles,

\footnotetext{
${ }^{12}$ Les entretiens concernant GAZACA ont été menés en mai 2009.
} 
comme par exemple entre la CCI et la $\mathrm{CGPME}^{13}$ locales. Tout ceci conduit à affirmer que, non seulement une agglomération d'entreprises (c'est-à-dire une concentration sur un périmètre géographique relativement restreint) n'assure pas, à elle seule, la qualité du relationnel entre ces organisations, mais que, de plus, cette même proximité géographique peut au contraire constituer une difficulté supplémentaire à les faire œuvrer ensemble sur un projet commun.

"Pour féconder des interactions, la proximité géographique doit être structurée et activée par la proximité organisée. » (Rallet \& Torre, 2004, p.5). La flexicurité constitue, nous semble-t-il, une situation particulièrement révélatrice de proximité organisée. Elle touche en effet à des aspects hautement stratégiques de la vie de l'organisation qui choisit de la mettre en place, avec des conséquences fortes en termes d'image comme acteur économique local. Elle exige à la fois un travail amont de qualité mais aussi une mise en œuvre adéquate, qui passe par une collaboration sublimée. Par collaboration, nous considérons les deux dimensions indissociables de coordination, qui structure le projet, et la coopération, ajustement mutuel traduisant la volonté, pour les acteurs impliqués, d'avancer ensemble (Defélix et al., 2008). S'y ajoute une dimension de solidarité (Le Boulaire \& Leclair, 2006), caractérisée par une recherche de souplesse qui se traduit par une flexibilité interne systématiquement privilégiée par rapport à une flexibilité externe, et par la nécessité de raisonner en réseau, en développant une « dynamique relationnelle [qui favorise] une sorte de développement social conjoint ». Le concept de solidarité ainsi mis en évidence nous semble caractériser de manière non équivoque la nature du réseau développé entre employeurs pour permettre à la flexicurité d'émerger. D'ailleurs, cette solidarité s'inscrit largement dans le concept de « coopération » précisé ci-dessus, comme une vision partagée. Ceci étant dit, cette solidarité ne se présume pas, comme en attestent les tentatives avortées de GAZACA ou de Saint-Flour Haute-Auvergne, et n'est pas acquise non plus, même sur un terrain a priori plus favorable, comme les GE du Sport, qui, en étant issus du milieu associatif, sont peut-être prédisposés à pratiquer une certaine solidarité. Mais, pour eux également, une concurrence existe entre les clubs adhérents (les créneaux d'activité étant par exemple identiques).

\subsection{Le niveau "méso », ou la flexicurité comme innovation sociale sur un territoire}

Notre recherche met en évidence le rôle crucial de plusieurs dimensions comme autant de conditions nécessaires (sans être pour autant suffisantes) à la flexicurité émergente. Ainsi, la proximité des différents acteurs, géographique et organisée, est indispensable, de même que la prise de conscience que chacun, employeurs comme salariés, peut avoir intérêt à mettre en pratique des dispositifs « gagnant-gagnant». Il semble ici intéressant de mobiliser la notion de milieu innovateur, entendu comme « un ensemble territorialisé dans lequel des interactions entre agents économiques se développent par l'apprentissage qu'ils font de transactions multilatérales génératrices d'externalités spécifiques à l'innovation et par la convergence des apprentissages vers des formes de plus en plus performantes de gestion en commun des ressources. »(Camagni \& Maillat, 2006, p.5).

\footnotetext{
${ }^{13}$ La CGPME du Puy-de-Dôme avait présenté une liste lors des élections consulaires de 2004, contre la liste sortante, qui a finalement été réélue.
} 
Dans le cas de la flexicurité émergente, les ressources considérées sont les hommes. La prise en compte du niveau «méso », allant au-delà des frontières traditionnelles de l'entreprise, n'est pas novateur en soi. Les travaux sur les districts industriels italiens ou sur les systèmes productifs locaux mettent clairement en avant l'intégration réticulaire et l'ancrage territorial (Carluer, 2006). Ce qui l'est davantage, c'est la capacité que peuvent avoir des employeurs à inventer des solutions nouvelles pour résoudre leurs problématiques d'emploi (flexibilisation mais aussi sécurisation), celles-ci étant tout aussi profitables à leurs salariés, comme au territoire sur lequel ils sont installés. Il s'agit donc d'une logique « gagnant-gagnant » à trois degrés.

\section{Conclusion}

Mettre en œuvre des pratiques de flexicurité émergente, telles que nous les avons observées, cela veut dire ne pas se satisfaire d'une gestion à courte vue (une entreprise raisonnant à court terme, pour elle-même), mais au contraire développer des collaborations, en l'occurrence avec d'autres entreprises, sur un même territoire. C'est ce que nous avons désigné par la proximité organisée. Nous avons précisé que cette collaboration passe aussi bien par la coordination des différents acteurs en vue d'atteindre un but commun (ici, la mise en œuvre de pratiques effectives de flexicurité) que par la coopération, c'est-à-dire la volonté d'aboutir. Nous avons ajouté une dimension de solidarité, qui nous semble indispensable à la réussite d'un tel projet. Sont donc concernés par cette proximité organisée : les entreprises du territoire, d'une part ; ces entreprises et leurs salariés actuels, d'autre part ; les entreprises et les salariés futurs, également ; et, enfin, le territoire, qui est ici un espace d'action, dans lequel tous les acteurs (entreprises, salariés, autres habitants, pouvoirs publics locaux, etc.) sont en interaction permanente. Ce dernier point illustre parfaitement le fait que «les territoires ne sont pas des champs de manøuvre, mais des acteurs. »(Veltz, 2005, p.157).

L'hypothèse initiale considérant le niveau " méso » s'avère effectivement pertinente, d'autant plus en s'intéressant aux PMO, dont le réseau est apparu à la fois construit, dans le cadre de projets collaboratifs faisant intervenir des intervenants multiples, mais aussi spontané, puisque la «construction » d'un projet, même bien faite, s'est avérée insuffisante. Cette idée de réseau existant préalablement, de manière formelle ou informelle, renvoie à la notion de milieu innovateur, " l'idée sous-jacente de la notion de milieu [étant] que les réseaux d'innovation vont se former à partir de relations déjà existantes. » (Maillat et al., 1991). Il nous semble donc particulièrement intéressant de revenir à la notion marshallienne d' " atmosphère industrielle », dans sa conception d'origine. Ainsi, les PMO, installées sur un territoire donné, mettent en œuvre une coopération stimulante visant à mettre en place des dispositifs concrets et efficaces de flexicurité, induisant en cela un développement vertueux qui impacte leur environnement, partenaires comme territoire. Elles créent alors, à l'instar de l'analyse marshallienne des villes de Sheffield ou Solingen (Marshall, 1919) un contexte favorable, terreau unique non transférable, propice à l'émergence de pratiques de flexicurité réussie.

\section{Références}

Auer P., Gazier B., 2006, L'introuvable sécurité de l'emploi, Flammarion, Paris. 
Avenier M.-J., 2004, « L'élaboration de savoirs actionnables en PME légitimés dans une conception des sciences de gestion comme des sciences de l'artificiel », Revue Internationale PME 17 (3-4), 13-42.

Barbier J.-C., 2009. Workfare, "flexicurité », réflexions sur le voyage international des idées politiques dans les politiques sociales et la persistance des compromis nationaux insérés dans les cultures politiques nationales. Congrès de l'AFSP, Grenoble.

Beaucourt C., 2008, Les niveaux d'équilibre de la flexicurité, XIX ${ }^{\mathrm{e}}$ Congrès de l'AGRH, Dakar.

Bernon J., Grillet S., Quint C., Trouvé P., 2006, La mutualisation des ressources humaines pour des TPE localisées sur un territoire, XVIIe Congrès de l'AGRH, Reims.

Boissonnat J., 1995, Le travail dans vingt ans, Editions Odile Jacob, Paris.

Bouba-Olga O., Grossetti M., 2008, « Socio-économie de proximité », Revue d'Economie Régionale et Urbaine $3,311-328$

Bouba-Olga O., Zimmermann J.-B., 2004, « Modèles et mesures de la proximité », in Pecqueur B. et Zimmermann J.-B. (dir.), Economie de proximités, Hermès Lavoisier, p.89-111

Boyer R., 2006, La flexicurité danoise : quels enseignements pour la France ? Collection du CEPREMAP, Presses de l'ENS.

Brandenburger A., Nalebuff B. , 1997, Co-opetition : a revolutionary mindset that combines Competition and Cooperation, Currency

Bredgaard, T., Larsen F., Madsen P.K., 2005, "The Flexible Danish Labour Market - A Review”, Aalborg: CARMA Research Paper 2005:01.

Bunel M., 2004, « Arbitrage entre flexibilité interne et flexibilité externe : une analyse empirique », Documents d'Etudes, DARES, n81.

Butera F., 1991, La métamorphose de l'organisation, du château au réseau, Les Editions d'Organisation, Paris.

Cahuc P., Kramarz F., 2004, De la précarité à la mobilité : vers une Sécurité Sociale professionnelle, Rapport au Ministre de l'Emploi, du Travail et de la Cohésion Sociale.

Camagni R., Maillat D. (coord.), 2006, Milieux innovateurs, théorie et politiques, Economica Anthropos, Paris.

Carluer F., 2006, « Réseaux d'entreprise et dynamiques territoriales : une analyse stratégique », Géographie, Economie, Société 8(2), 193-214.

CERC, 2005, La sécurité de l'emploi face aux défis des transformations économiques, La documentation française, Paris.

Defélix C., Klarsfeld A., Oiry E. (coord.), 2006, Nouveaux regards sur la gestion des compétences, Vuibert, Paris.

Defélix C., Colle T., Rapiau M.-T., 2008, « Prendre en compte le facteur humain au sein des pôles de compétitivité : la longue marche vers l'innovation sociale », Management et Avenir, n²0, novembre, p.9-29.

Eisenhardt K. M., 1989, "Building Theories from Case Study Research", Academy of Management Review 14(4), 532-550.

Everaere C., 2008, « La polyvalence et ses contradictions », Revue Française de Gestion Industrielle 27(4), 89-104.

Fabre E., De Riccardis N., 2007, « Les contrats courts vus par les salariés : une précarité de l'emploi qui n'induit pas nécessairement une précarité du travail », Premières Informations, Premières Synthèses, DARES, ${ }^{\circ} 12.3$

Fourcade C., 2008, « Des dynamiques territorialisées novatrices : le cas des PME agro-alimentaires » Revue d'Economie Régionale et Urbaine 2, 193-210.

Gavard-Perret M.-L., Helme-Guizon A., 2008, "Choisir parmi les techniques spécifiques d'analyse qualitative", in Gavard-Perret M.-L., Gotteland D., Haon C., Jolibert A. (dir.) Méthodologie de la recherche. Réussir son mémoire ou sa thèse en sciences de gestion. Pearson Education, pp.247-279.

Gazier B., 2003, Tous « sublimes », vers un nouveau plein-emploi, Flammarion, Paris.

Gazier B., 2006, «L'employabilité », in Allouche J. (coord.), Encyclopédie des Ressources Humaines, $2^{\mathrm{e}}$ édition, Vuibert, pp. 350-357. 
Gilly J.-P., Lung Y., 2005, « Proximités, secteurs et territoires », in Laurent C., Du Tertre C., Secteurs et territoires dans les régulations émergentes, L'Harmattan, pp. 161-180.

Girin J., 1990, « L'analyse empirique des situations de gestion : éléments de théorie et de méthode » in Martinet A.-C. (coord.), Epistémologies et Sciences de Gestion. Economica, pp.141-182.

Hlady Rispal M., 2002, La méthode des cas, application à la recherche en gestion, De Boeck Université, Bruxelles.

Igalens J., 2006, « Evolution de la division du travail : du métier au réseau ». In Allouche J. (coord.), Encyclopédie des Ressources Humaines, $2^{\mathrm{e}}$ édition, Vuibert, pp. 295-297.

Jørgensen, H., Madsen P.K., 2007, "Flexicurity and Beyond - Reflection on the Nature and future of a Political Celebrity, Jørgensen H., Madsen P.K., (eds.) Flexicurity and Beyond - Finding a New Agenda for the European social Model, Copenhagen: DJOEF Publishing, pp. 7-39.

Le Boulaire M., Leclair P., 2006, « Entreprise-réseau et gestion des ressources humaines », in Allouche J. (coord.), Encyclopédie des Ressources Humaines, 2édition, Vuibert, pp. 1136-1146.

Leschke J., Schmid G., Griga D., 2006, "On the marriage of flexibility and security : lessons from the Hartz reforms un Germany”, WZB Discussion Papers, SP I 2006-108.

Madsen P. K., 2004, “The Danish model of "flexicurity": experiences and lessons", Transfer, 10 (2), 187-207.

Maillat D, Crevoisier O., Lecoq B., 1991, « Réseaux d'innovation et dynamique territoriale : un essai de typologie », Revue d'Economie Régionale et Urbaine 3-4, 407-432.

Marchesnay M., Fourcade C. (dir.), 1997, Gestion de la PME/PMI, Nathan, Paris.

Marshall A., 1919, Industry and Trade, Macmillan, traduction française : L'industrie et le commerce, Giard, 1934.

Méda D., Minault B., 2005, « La sécurisation des trajectoires professionnelles », Documents d'Etudes DARES $\mathrm{n}^{\circ} 107$.

Messeghem K., Paradas A., 2008, «Pôles de compétitivité et relations amont/aval : entre pouvoir et légitimité », in Gundolf K., Jaouen A. (dir.), Les relations interorganisationnelles des PME, Hermès Lavoisier, pp.223-248.

Pecqueur B., 2009, «De l'exténuation à la sublimation : la notion de territoire est-elle encore utile ? » Géographie, Economie, Société 11(1), 55-62.

Pichault F., Xhauflair V., 2007, La flexsécurité revisitée à l'aune des pratiques effectives, XVIIIe congrès de l'AGRH, Fribourg.

Pichault F., Xhauflair V., 2009, Flexibility and Security : micro perspectives on dealing with Flexicurity, International Conference and Doctoral Consortium on evaluation metrics of corporate social and environmental, ISEOR/Academy of Management, Lyon.

Rallet A., Torre A., 2004, « Proximité et localisation », Economie rurale 280 mars/avril, 25-41.

Supiot, A., 1999, Au-delà de l'emploi : transformation du travail et devenir du droit du travail en Europe, rapport pour la Commission Européenne, Flammarion, Paris.

Torrès O., 2007, «Approche descriptive de la spécificité de gestion des PME : le mix de proximité », in Filion L.-J. (dir.), Management des PME, de la création à la croissance, Pearson Education, pp. 23-46.

Veltz P., 2005, Mondialisation, villes et territoires, PUF, Paris.

Wilthagen T., Rogowski R., 2002, "Legal regulation of transitional labour markets", in Schmid G., Gazier B. (dir.). The dynamics of full employment : social integration through transitionnal labour markets, Edward Elger, pp.233-273.

Wilthagen T., Tros, F., 2003, The concept of «Flexicurity » : A new approach to regulating employment and labour market,

http://www.tilburguniversity.nl/faculties/law/research/flexicurity/publications/papers/fxp2003_4.pdf

Zarifian P., 1999, Objectif compétence, Editions Liaisons, Paris.

Zimmermann J.-B., 2008, « Le territoire dans l'analyse économique. Proximité géographique et proximité organisée », Revue Française de Gestion 184, 105-118. 
